\title{
Lung ultrasound monitoring of CPAP effectiveness on SARS-CoV-2 pneumonia: A case report
}

\author{
Marco Montanari, ${ }^{1}$ Pierpaolo De Ciantis, ${ }^{1}$ Andrea Boccatonda, ${ }^{2,3}$ Marta Venturi, ${ }^{1}$ \\ Giuseppe d'Antuono, ${ }^{1}$ Gianfilippo Gangitano, ${ }^{1}$ Giulio Cocco,${ }^{3}$ Damiano D'Ardes, ${ }^{3}$ \\ Cosima Schiavone, ${ }^{3}$ Fabrizio Giostra, ${ }^{2}$ Tiziana Perin ${ }^{1}$ \\ ${ }^{1}$ Medicina d'Urgenza e Pronto Soccorso, Ospedale Infermi, Rimini; ${ }^{2}$ Medicina d'Urgenza e Pronto \\ Soccorso, Policlinico Sant'Orsola-Malpighi, Azienda Ospedaliero-Universitaria di Bologna; ${ }^{3}$ UOSD \\ Ecografia Internistica, “G. d'Annunzio” University, Chieti, Italy
}

\begin{abstract}
SARS-CoV-2 infection is characterized by extremely heterogeneous features, going from cases with few symptoms to severe respiratory failures. Chest Computed Tomography (CT) is currently the gold-standard imaging method, although burdened by the risk of exposure to ionizing radiation and management / organizational concerns. In particular, the critical patient undergoing ventilation (invasive or not) seems to be difficult to monitor by repeated CT scan over time. We report the case of a 55-year-old male patient subjected to Continuous Positive Airway Pressure (CPAP) and prone positioning, in which the use of ultrasound monitoring allowed to verify the effectiveness of the pressure support used in recruiting previously atelectasis lung areas. Lung ultrasound can guide pulmonary recruitment and pronation maneuvers in patients undergoing non-invasive ventilation. Ultrasound can identify atelectatic lung areas, which demonstrate an alveolar re-expansion following the setting of high PEEP values, as underlined by the reappearance of pleural/air interface.
\end{abstract}

Correspondence: Andrea Boccatonda, Policlinico Sant'Orsola-
Malpighi, via Pietro Albertoni, 15, 40138 Bologna BO, Italy
Tel.: +390512141111
E-mail: andrea.boccatonda@aosp.bo.it
Key words: Ultrasound; Covid-19; pneumonia; CPAP; ventilation;
prone position.
Ethics approval and consent to participate: informed consent was
obtained from the patient.
Consent for publication: Informed consent was obtained from the
patient
Conflict of interest: No one. This work was not supported by any grant.
Received for publication: 1 September 2020.
Accepted for publication: 6 November 2020.
This work is licensed under a Creative Commons Attribution 4.0
License (by-nc 4.0 ).
CCopyright: the Author(s), 2020
Licensee PAGEPress, Italy
Emergency Care Journal $2020 ; 16: 9333$
doi:10.4081/ecj.2020.9333

\section{Introduction}

The SARS-CoV-2 pandemic has surprisingly brought to light the role of lung ultrasound. ${ }^{1,2}$ Covid-19 infection is characterized by extremely heterogeneous features, going from cases with few symptoms to severe respiratory insufficiencies and fatal complications. ${ }^{3-5}$ Chest Computed Tomography (CT) is currently the goldstandard imaging method offering the best diagnostic accuracy, although burdened by the risk of exposure to ionizing radiation and management / organizational concerns. ${ }^{6}$ In particular, the critical patient undergoing ventilation (invasive or not) seems to be difficult to monitor by repeated CT scan over time. Lung ultrasound is a bed-side, simple, low cost and repeatable method., ${ }^{1,2}$ Some reports in literature reported that awake early proning in the emergency department induces improved oxygen saturation in Covid19 patients. ${ }^{7,8}$ We report the case of a 55 -year-old male patient hospitalized in a critical setting and subjected to Continuous Positive Airway Pressure (CPAP) and prone position, in which the use of ultrasound monitoring allowed to verify the effectiveness of the pressure support used in recruiting previously atelectasis lung areas.

\section{Case Report}

A 55-year-old male patient was transferred to our unit from a spoke hospital for worsening respiratory failure treated with conventional oxygen therapy. The patient had been hospitalized for the onset of dyspnea, fever and cough from about 10 days. His past medical history was characterized by hypertension treated with valsartan/ hydrochlorothiazide, first degree obesity. In the medical history, there were no pulmonary disease and no tobacco consumption. The patient weighed $94 \mathrm{~kg}$ and was $173 \mathrm{~cm}$ tall. Blood exams revealed: white blood cells: 10.29 x109/L (4.00 - 10.00), hemoglobin $13.3 \mathrm{~g} / \mathrm{dL}$ (13.5 - 17.0), neutrophils $9.10 \times 10^{9} / \mathrm{L}$ (2.00 - 8.00), lymphocytes $0.74 \times 10^{9} / \mathrm{L}(1.00$ - 4.00), D-dimer $813 \mathrm{ug} / \mathrm{L}$ (Cut-off VTE exclusion $<500)$, creatinine $0.64 \mathrm{mg} / \mathrm{dL}(0.70-1.20)$, AST $251 \mathrm{U} / \mathrm{L}(<40)$, ALT 349 U/L (<41), LDH 950 U/L (135 - 225), C-reactive protein $2,2 \mathrm{mg} / \mathrm{L}(<5.0)$, high-sensitivity-troponin 9 ng/L (99th percentile: $15 \mathrm{ng} / \mathrm{L})$, procalcitonin $0.9 \mathrm{ug} / \mathrm{L}(<0,5$ low risk of sepsis), IL-6 $20.7 \mathrm{pg} / \mathrm{ml}(<5.9)$, PT/INR 1.33 (0.8 - 1.2). Blood cultures were negative for bacterial and fungal growth.

Bilateral parenchymal consolidations were present on the chest $\mathrm{X}$-ray, more evident in the upper lobes, minimal bilateral pleural effusion and normal cardio-mediastinal shadow. A SARS-CoV-2 positive swab was performed. On the same day, he carried out infectious disease advice recommending antiviral therapy with darunavir $800 \mathrm{mg} 1 \mathrm{cp} \mathrm{OD}$ and ritonavir $100 \mathrm{mg} 1 \mathrm{cp} \mathrm{OD}$ and 
hydroxychloroquine $200 \mathrm{mg} 1 \mathrm{cp} \mathrm{BD}$. Furthermore, dexamethasone (20 mg OD) and low molecular weight heparin (LMWH) (6000 UI OD) therapy was undertaken. Then, the patient was transferred to our emergency medicine ward for clinical worsening. Vital parameters were: Respiratory Rate (RR) 30 acts/min, cardiac rate $100 \mathrm{bpm}$, blood pressure 140/70 $\mathrm{mmHg}$, peripheral oxygen saturation $94 \%$ on conventional oxygen therapy $15 \mathrm{liter} / \mathrm{min}(\mathrm{P} / \mathrm{F}$ $=100$ ). Lung ultrasound performed during conventional oxygen therapy showed the presence of bilateral sub-pleural consolidations, with some lung fields characterized by coalescent B lines as for "white lung" (Figure 1); taking as reference the Lung Ultrasound Score9 exam scheme (Figure 2), the patient was given a value of 32 .

In the emergency medicine ward, the patient was first treated with Ventimask (Fio2: 60\%) reporting a P/F value of 115 . Then, the patient was subjected to a protocol based on CPAP with helmet interface (StarMEd helmet interface) with flow $85 \mathrm{~L} / \mathrm{min}(14+$ 14), $\mathrm{FiO} 257 \%$ and PEEP $12.5 \mathrm{~cm} \mathrm{H}_{2} \mathrm{O}$. The patient was subsequently positioned in the prone position to obtain a further improvement in gas exchange. The improvement of the Arterial Blood Gas Analysis (ABG) data was highlighted after four hours (Table 1). In particular, the patient had an improvement of $\mathrm{P} / \mathrm{F}$ (215), and a reduction of the RR of about 5 acts/min during pronation (see Table 1 for comparison). The therapeutic protocol required the patient to perform CPAP in the prone position during the day, and in the supine position during the night.

After two days of CPAP treatment and prone positioning, the consolidative areas disappeared and was documented the presence of areas with non-coalescent $B$ lines and even lung fields with pattern A; the LUS score was 4 (Figure 3). The alveolar gas exchanges always remained constant over time of CPAP therapy. The patient performed the CPAP and pronation protocol for 5 days. The possibility of continuous assistance from the staff and the good compliance of the patient did not require the use of sedative therapies to perform CPAP.

\section{Discussion}

SARS-Cov-2 can induce pneumocytic damage directly and generate an inflammatory response leading to an accumulation of inflammatory fluid that occupies the interstitium and progressively the alveolus; that induces a progressive loss of air which is progressively replaced by cellular / inflammatory material., ${ }^{5,10}$ On lung ultrasound, the progressive loss of alveolar aeration is manifested by the passage from a pleural air interface with A lines (horizontal artifacts) to one with B lines (vertical artifacts), which gradually increase in number and become coalescent (white lung), ${ }^{2,11-14}$ Previous data demonstrated a correspondence between the ultrasound finding of white lung and that of ground-glass on chest CT. ${ }^{1,2}$ In the most severely affected areas, the total loss of alveolar air manifests on ultrasound with a solid pattern, thus showing lung consolidations, with the presence of any aerial bronchograms. Moreover, in the critically ill patient with severe hypoxemia and alteration of the respiratory drive, areas of atelectasis due to basal fields disventilation may occur, due to diaphragmatic hypomobility, thus leading to worsening of the clinical picture ${ }^{15,16}$

This process of progressive alveolar de-aeration finds a reverse confirmation through to the use of ventilation methods. In our case, a CPAP therapy with prone positioning was used, that allowed the re-ventilation of atelectasis areas through the application of positive pressure. ${ }^{17}$ In pneumonia, CPAP was used mainly for non-hypercapnic acute respiratory failure. ${ }^{18-20}$ On ultrasound, the process of re-ventilation is evidenced by the progressive disappearance of the consolidation areas, in favor of an air interface, with a gradual reduction in the number of B lines, up to a normal A pattern. Therefore, lung ultrasound allows to monitor the effect

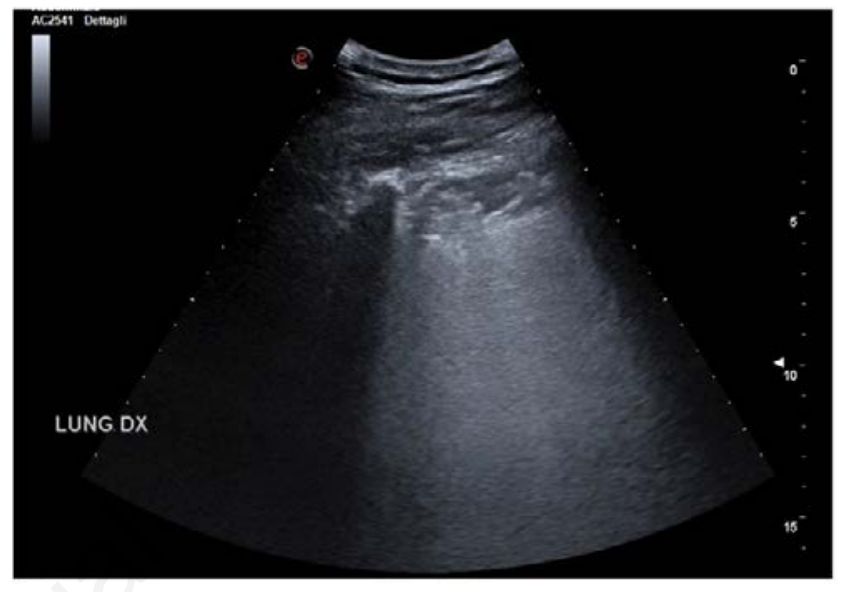

Figure 1. Lung ultrasound on conventional oxygen therapy: bilateral subpleural consolidations, with some lung fields characterized by coalescent B lines as for "white lung".

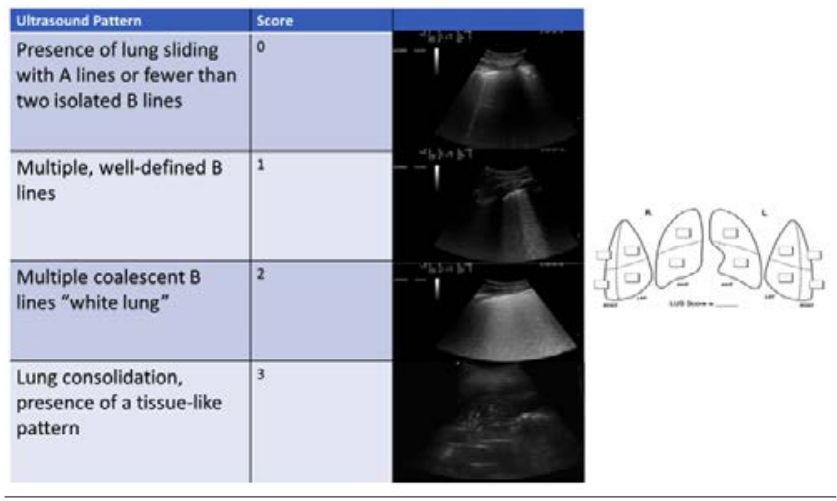

Figure 2. Ultrasound scan was taken in six regions for each lung (superior and inferior areas in the anterior, lateral, and posterior fields using anterior and posterior axillary lines as landmarks). Four ultrasound aeration patterns were defined in each region: presence of lung sliding with $A$ lines or fewer than two isolated $B$ lines (0); multiple, well-defined B lines (1); multiple coalescent B lines or white lung (2); lung consolidation (3). LUS scores were calculated as the sum of all.

Table 1. ABG values and Respiratory Rate (RR) in the different phases of oxygen therapy and non-invasive ventilation.

\begin{tabular}{lcccccc} 
& $\mathrm{pH}$ & $\mathrm{pO}_{2}(\mathrm{mmHg})$ & $\mathrm{pCO} 2(\mathrm{mmHg})$ & $\mathrm{P} / \mathrm{F}$ & $\mathrm{FHO}_{2}(\%)$ & $\mathrm{RR}(\mathrm{acts} / \mathrm{min})$ \\
Ventimask & 7,53 & 69,3 & 28 & 115 & $60 \%$ & 36 \\
CPAP & 7,47 & 80 & 26 & 140 & $57 \%$ & 33 \\
\hline CPAP + prone position & 7,48 & 123 & 32 & 215 & $57 \%$ & 28 \\
\hline
\end{tabular}




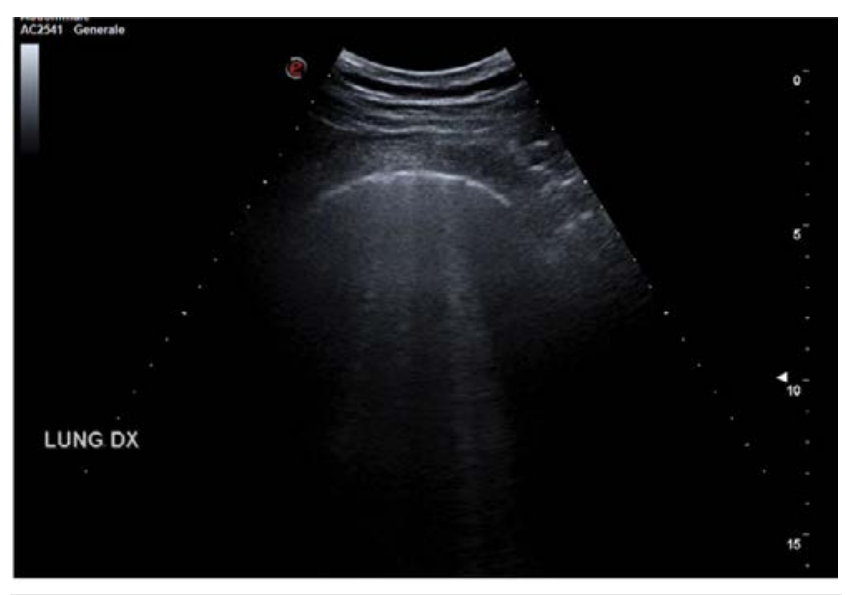

Figure 3. Lung ultrasound after CPAP: non-coalescent B lines and even lung fields with pattern $A$.

of applying a ventilation method (positive pressure) on specific lung areas, scanned in a serial way. Furthermore, the verification of the success of the attempt to re-expand atelectasis lung areas allows to set the pressure values employed by CPAP more precisely. In our case, pronation maneuver was also performed, thus leading to good results even with non-invasive ventilation.

In our opinion, the improvement of pulmonary compromise, as demonstrated by ultrasound, is due to CPAP and pronation therapy rather than to a healing process of the pathology, since the time elapsed between the two checks ( 2 days) is too much short, and in this period of time no specific therapies were included.

\section{Conclusions}

It is possible to monitor SARS-CoV-2 pneumonia evolution by ultrasound through a seriated evaluation of signs of pulmonary disease (B lines reduction / increase or appearance / disappearance of the consolidations and atelectasis) by adopting an ultrasound execution scheme. Lung ultrasound can guide pulmonary recruitment and pronation maneuvers in patients undergoing invasive ventilation; indeed, ultrasound can identify atelectatic lung areas, which demonstrate an alveolar re-expansion following the setting of high PEEP values, as underlined by the reappearance of pleural air interface.

\section{References}

1. Poggiali E, Dacrema A, Bastoni D, et al. Can lung US help critical care clinicians in the early diagnosis of novel coronavirus (Covid19) Pneumonia? Radiol 2020;13:200847. doi:10.1148/radio 1.2020200847.

2. Sofia S, Boccatonda A, Montanari M, et al. Thoracic ultrasound and SARS-Covid-19: a pictorial essay. J Ultrasound 2020;16:1-5. doi:10.1007/s40477-020-00458-7.

3. Huang C. Clinical features of patients infected with 2019 novel coronavirus in Wuhan. China Lancet 2020;395:497-506. doi:10. 1016/S0140 -6736(20)30183-5

4. Weiss P, Murdoch DR. Clinical course and mortality risk of severe Covid-19. Lancet 2020;395:1014-5. doi:10.1016/S01406736(20)30633-4
5. D'Ardes D, Boccatonda A, Rossi I, et al. Covid-19 and RAS: Unravelling an Unclear Relationship. Int J Mol Sci 2020;21:3003. doi:10.3390/ijms21083003

6. Shi H, Han X, Jiang N, et al. Radiological findings from 81 patients with Covid-19 pneumonia in Wuhan, China: a descriptive study. Lancet Infect Dis 2020;20: 425-34. doi:10.1016/S14733099(20)30086-4

7. Caputo ND, Strayer RJ, Levitan R. Early Self-Proning in Awake, Non-intubated Patients in the Emergency Department: A Single ED's Experience During the Covid-19 Pandemic. Acad Emerg Med 2020;27:375-8.

8. Bastoni D, Poggiali E, Vercelli A, et al. Prone positioning in patients treated with non-invasive ventilation for Covid-19 pneumonia in an Italian emergency department. Emerg Med J 2020;37:565-6.

9. Bouhemad B, Mojoli F, Nowobilski N, et al. Use of combined cardiac and lung ultrasound to predict weaning failure in elderly, highrisk cardiac patients: a pilot study. Intensive Care Med 2020;46: 475-84

10. Bao L, Deng W, Huang B, et al. The pathogenicity of SARS-CoV2 in hACE2 transgenic mice. Nature 2020;583:830-3. doi:10.1038/ s41586-020-2312-y

11. Reissig A, Copetti R. Lung ultrasound in community acquired pneumonia and in interstitial lung diseases. Respiration 2014;87:179-89. doi:10.1159/000357449

12. Boccatonda A, Primomo G, Cocco G, et al. Not all abolished lung sliding are pneumothorax: the case of a particular lung atelectasis. J Ultrasound 2020. doi:10.1007/s40477-020-00427-0

13. Laursen CB, Sloth E, Lassen AT, et al. Point-of-care ultrasonography in patients admitted with respiratory symptoms: a single-blind, randomised controlled trial. Lancet Respir Med 2014;2:638-46.

14. Boccatonda A, Ianniello E, D'Ardes D et al. Can lung ultrasound be used to screen for pulmonary embolism in patients with SARSCoV-2 pneumonia? Eur J Case Rep Intern Med 2020;7:001748. doi:10.12890/2020_001748

15. Copetti R, Soldati $\bar{G}$, Copetti P. Chest sonography: a useful tool to differentiate acute cardiogenic pulmonary edema from acute respiratory distress syndrome. Cardiovascular Ultrasound 2008. PROVIDE A LINK

16. Mongodi S, Pozzi M, Orlando A, et al. Lung ultrasound for daily monitoring of ARDS patients on extracorporeal membrane oxygenation: preliminary experience. Intensive Care Med 2018;44:123-4. doi:10.1007/s00134-017-4941-7

17. Généreux V, Chassé M, Girard F, et al. Effects of positive end-expiratory pressure/recruitment manoeuvres compared with zero endexpiratory pressure on atelectasis during open gynaecological surgery as assessed by ultrasonography: a randomised controlled trial. Br J Anaesth 2020;124:101-9. doi:10.1016/j.bja.2019.09.040.

18. Brambilla AM, Prina E, Ferrari G, et al. Non-invasive Positive Pressure Ventilation in Pneumonia Outside Intensive Care Unit: An Italian Multicenter Observational Study. Eur J Intern Med 2019;59:21-6. doi:10.1016/j.ejim.2018.09.025

19. Lari F, Giostra F, Bragagni G, et al. Continuous positive airway pressure in acute pulmonary edema. application in a general medical ward]. Recenti Prog Med 2011;102:114-9. doi:10.1701/608. 7067

20. Nava S, Carbone G, Di Battista N, et al. Noninvasive ventilation in cardiogenic pulmonary edema: a multicenter randomized trial. Am J Respir Crit Care Med 2003;15;168:1432-7. doi:10.1164/rccm. 200211-1270OC 